\title{
ISCHEMIA questions and MITNEC answers: Defining and standardizing clinical ischemic jeopardy with SPECT myocardial perfusion imaging
}

\author{
Erin M. Armenia, BA, MD, and Ronald G. Schwartz, MD, MS, MASNC ${ }^{\mathrm{a}}$ \\ a University of Rochester Medical Center, Rochester, NY
}

Received May 9, 2020; accepted May 11, 2020

doi: $10.1007 / \mathrm{s} 12350-020-02237-7$

See related article, pp. 2712-2725

Cardiac imaging of the extent and severity of myocardial ischemia has revolutionized the diagnosis, risk assessment and treatment of ischemic heart disease. Radionuclide perfusion imaging with SPECT and PET provides safe, incremental diagnostic, and prognostic assessments and the ability to monitor treatment response and inform clinical decisions of therapeutic options of residual risk in patients with suspected, stable, and/or unstable ischemic heart disease ${ }^{1-24}$ In addition to identifying relative degrees of ischemia and culprit stenosis in multivessel disease, numerous studies have demonstrated prognostic assessment for hard clinical events of death and/or myocardial infarction of SPECT and PET MPI employing semi-quantified regional perfusion defects or quantification of myocardial flow reserve meets or exceeds substantially that of exercise ECG or angiographic CAD and identify patients who benefit from CABG rather than PCI. ${ }^{1-6,14-16}$ This prognostic power reflects the integrated determinants of flow of the entire coronary tree including focal stenoses, diffuse atherosclerotic luminal narrowing which can be under-estimated by arteriography and trans-stenosis fractional flow measurements, endothelial vasomotor dysfunction associated with atherosclerotic coronary plaque, and the microvasculature. ${ }^{10-18}$

Reprint requests: Ronald G. Schwartz, MD, MS, MASNC, University of Rochester Medical Center, Rochester, NY; ronald_schwartz@ urmc.rochester.edu

J Nucl Cardiol 2021;28:2726-9.

$1071-3581 / \$ 34.00$

Copyright (c) 2020 American Society of Nuclear Cardiology.
Extent and severity of stress LV myocardial perfusion defects and ischemia scores on radionuclide MPI contribute to prognostic value of coronary event risk. ${ }^{25}$ Segmental LV models of summed stress (SSS), rest (SRS) scores, and difference scores (SDS) that reflect degree of ischemia using a 5-point segmental perfusion score became standardized employing 20 segments initially. ${ }^{5}$ The 17 -segment LV model was adapted as an optimally weighted approach for interpreting SPECT MPI studies. ${ }^{26}$ Current ASNC guidelines recommend both qualitative and quantitative reporting of perfusion defects. ${ }^{27}$ Qualitative reporting involves identifying the affected area based on a 17-segment polar plot, and classifying the defect as mild, moderate, or severe based on the number of involved segments. Quantitative defect assessment per the ASNC guidelines involves scoring each of the $17 \mathrm{LV}$ myocardial segments on a 5-point scale from 0 to $4(0=$ no ischemia, $1=$ mild or equivocal, $2=$ moderate, 3 = severe, $4=$ absence of perfusion), summing the stress scores (SSS) and rest scores (SRS), and calculating the summed difference score (SDS). Total perfusion deficit (TPD) percentages can subsequently be calculated by dividing SSS, SRS, and SDS into the theoretical maximum hypoperfusion score which is 68 for a 17 -segment model. ${ }^{27}$

This methodology has been widely implemented in clinical practice and in outcome studies of SPECT and PET MPI. Hachamovitch demonstrated substantial incremental risk assessment with SSS and SDS in low-, intermediate-, and high-risk categories as defined by the Duke exercise treadmill test. ${ }^{3}$ Large observational studies have shown short-term benefit of revascularization compared to medical therapy became apparent with more than $10 \% \mathrm{LV}$ ischemia and became statistically significant at a level of $20 \% \mathrm{LV}$ ischemia, a benefit that could be offset by the presence of large $(>10 \% \mathrm{LV})$ myocardial scar. ${ }^{5,6}$ Early observational PET and SPECT 
MPI studies demonstrated reductions of stress perfusion defect size and severity scores 1-6 months in response to statin and lifestyle therapies of CAD ${ }^{21-24}$ correlated or anticipated the 6-12 months clinical time course of therapeutic response of major clinical statin trials. ${ }^{28}$ The nuclear cardiology sub-study of the randomized COURAGE trial observed ischemia reduction on serial SPECT MPI studies improved in both optimal medical therapy and revascularization arms of the trial. ${ }^{20}$

The recently published ISCHEMIA trial has challenged our concept of scintigraphic definition of ischemic jeopardy ${ }^{29}$ with the surprising finding of lack of correlation of the reported degree of ischemia by SPECT MPI and cardiac outcomes by 3 years of followup. No relationship was seen between ischemic severity and all-cause mortality and only a weak trend emerged for a relationship with 4-year cumulative rates of myocardial infarction. While CTA exclusion of left main disease and efficacy of optimal medical therapy likely reduced clinical event risk in the ISCHEMIA study patients, a major question posed by the ISCHEMIA trial is whether overestimation of ischemia extent and severity by recruiting site laboratories compared to the core lab biased the analyses by referring a relatively low-risk population. Standards for accuracy and reproducibility of perfusion defect quantification of SPECT MPI studies in community and core laboratories in the design and conduct of multi-modality clinical trials are required. Modality-specific performance measures will determine their level of diagnostic and prognostic value in the management and cost-effective development of new pharmacologic and therapeutic interventions to treat ischemic heart disease. Understanding the accuracy and precision of perfusion defect scoring will facilitate study design to monitor premorbid therapeutic response and reduce development costs of new pharmaceutical agents to treat ischemic heart disease.

In this issue of the Journal, the Medical Imaging Trials Network of Canada (MITNEC) reports its standardization and quality assurance program to develop state-of-the-art SPECT camera qualification and clinical interpretation standards to enable and ensure unbiased evaluation of the accuracy of the well-established technique of SPECT MPI vs. the alternative imaging modalities. ${ }^{30}$ Due to the large sample size of patient imaging data $(\mathrm{N}=600$ scans), two core labs were established to interpret the phantom and patient scans. The authors report highly repeatable quantitative scoring and interpretation of perfusion images was achieved between 2 core labs following standardization of Tc99m-SPECT MPI acquisition protocols and image interpretation. Rest-stress Tc-99m-SPECT phantom scans were acquired on 35 cameras. MPI defects were quantified with summed stress (SSS) and difference scores (SDS) at 2 core labs. $\mathrm{SDS} \geq 2$ in the right coronary artery (RCA) was the qualifying standard, and 20 rest $(\mathrm{R})$-stress $(\mathrm{S})$ patient images were acquired on qualified cameras and interpreted by core labs. Using phantoms, visual scoring was identified on all cameras. With patient scans $90 \%$ of global SSS and $85 \%$ of SDS differences were $\leq 3$. Correlation of regional analysis of SSS, SDS, more than mild global myocardial perfusion abnormalities (SSS $\geq 4$ ), and any ischemic abnormality (SDS $\geq 2$ ) was excellent, and clinical interpretation agreement was $100 \%$ following review. Automated analyses of ischemic total perfusion deficit (TPD), reversible TPD, transient ischemic dilation, and S-R LV ejection fraction were excellent. The authors conclude quantitative scoring and interpretation of scans was highly repeatable with site qualification and clinical interpretation standardization. The investigators demonstrated feasibility and appropriateness of dualcore lab interpretation to determine 99mTc-SPECT MPI accuracy for multi-center clinical trial scans.

The study design is robust, and several key strengths of the study merit appreciation. (1) Acquisition protocols and image interpretation were standardized, including the use of discrete color scales of image slices and polar plots to facilitate accurate and reproducible segmental quantification of regional perfusion, as illustrated in Figure 3 of the paper. ${ }^{17}$ (2) Studies of both phantom and patient studies confirm the high accuracy and reproducibility of the methodology. Phantom design realistically simulated patient scans by utilizing a 2:1 liver:heart activity concentration to simulate scatter from the liver and/or bowel activity. (3) The study was sufficiently powered with a sample size of 20 to permit detection of a clinically relevant SDS difference of 1 scoring unit, similar to the sample size used in their previously published PET paper that evaluated MPI scoring differences between readers. ${ }^{31}$ (4) The scan observations were performed by two senior nuclear cardiology physicians, both with over 20 years clinical experience reporting SPECT perfusion studies in a tertiary-care academic hospital. These data suggest that there was no bias between the two observers. (5) Consensus review may be beneficial at the start and throughout a clinical imaging trial to standardize methods and improve consistency between core labs, particularly in patients with mild or borderline disease categories. (6) The pooling of the results of the two core labs with similar performance characteristics into one dataset for evaluation enhanced the feasibility of studying the large data sets. This study identified high accuracy and reproducibility of detecting small and large amounts of ischemia, transient ischemic dilation, and stress-rest LVEF differences with Tc-99m SPECT MPI which was readily achievable utilizing two core 
labs when effective standardization methods were implemented.

As we consider the analyses of the IMAGE Guide Registry and differences in ischemia quantification of recruiting site and core laboratories to better understand the findings and implications of the ISCHEMIA Trial, MITNEC study of Tc-99m SPECT MPI has helped advance the field of cardiac imaging by defining testing standards for MPI defect quantification and feasibility of state-of-the-art multi-core lab organization and interaction to handle large data sets and achieve unbiased, accurate, and reproducible measurement of scintigraphic ischemia to compare to other imaging modalities. MITNEC has paved the road with high standards for investigation of the quantification of myocardial ischemic jeopardy and enduring assessment of therapeutic response to guide clinical practice and optimize patient outcomes.

\section{References}

1. Iskandrian AS, Chae SC, Heo J, Stanberry CD, Wasserleben V, Cave V. Independent and incremental prognostic value of exercise single-photon emission computed tomographic (SPECT) thallium imaging in coronary artery disease. J Am Coll Cardiol 1993;22:665-70.

2. Pancholy SB, Fattah AA, Kamal AM, Ghods M, Heo J, Iskandrian AS. Independent and incremental prognostic value of exercise thallium single-photon emission computed tomographic imaging in women. J Nucl Cardiol 1995;2:110-6. https://doi.org/10.1016/ S1071-3581(06)80020-6.

3. Hachamovitch R, Berman DS, Kiat H, Cohen I, Cabico JA, Friedman $\mathrm{J}$, et al. Exercise myocardial perfusion SPECT in patients without known coronary artery disease: Incremental prognostic value and use in risk stratification. Circulation 1996;93:905-14.

4. Gibbons RJ, Hodge DO, Berman DS, Akinboboye OO, Heo J, Hachamovitch R, Bailey KR, Iskandrian AE. Long-term outcome of patients with intermediate-risk exercise electrocardiograms who do not have myocardial perfusion defects on radionuclide imaging. Circulation 1999;100:2140-5.

5. Hachamovitch R, Hayes SW, Friedman JD, Cohen IO, Berman DS. Comparison of the short-term survival benefit associated with revascularization compared with medical therapy in patients with no prior coronary artery disease undergoing stress myocardial perfusion single photon emission computed tomography. Circulation 2003;107:2900-7. https://doi.org/10.1161/01.CIR. 0000072790.23090 .41 .

6. Hachamovitch R, Rozanski A, Shaw LJ, Stone GW, Thomson LEJ, Friedman JD, Hayes SW, Cohen I, Germano G, Berman DS. Impact of ischaemia and scar on the therapeutic benefit derived from myocardial revascularization vs. medical therapy among patients undergoing stress-rest myocardial perfusion scintigraphy. Eur Heart J 2011;32:1012-24. https://doi.org/10.1093/eurheartj/eh q500.

7. Udelson JE, Beshansky JR, Ballin DS, Feldman JA, Griffith JL, Handler J, Heller GV, Hendel RC, Pope JH, Ruthazer R, Spiegler EJ, Woolard RH, Selker HP. Myocardial perfusion imaging for evaluation and triage of patients with suspected acute cardiac ischemia: A randomized controlled trial. JAMA 2002;288:2693700. https://doi.org/10.1001/jama.288.21.2693.

8. Dorbala S, Giugliano RP, Logsetty G, et al. Prognostic value of SPECT myocardial perfusion imaging in patients with elevated cardiac troponin I levels and atypical clinical presentation. J Nucl Cardiol 2007;14:53-8. https://doi.org/10.1016/j.nuclcard.2006.07. 010.

9. Cremer PC, Khalaf S, Agarwal S, Mayer-Sabik E, Ellis SG, Menon $\mathrm{V}$, et al. Myocardial perfusion imaging in emergency department patients with negative cardiac biomarkers yield for detecting ischemia, short-term events, and impact of downstream revascularization on mortality. Circ Cardiovasc Imaging 2014;7:912-9.

10. Ludmer PL, Selwyn AP, Shook TL, Wayne RR, Mudge GH, Alexander RW, Ganz P. Paradoxical vasoconstriction by acetylcholine in atherosclerotic coronary arteries. N Engl J Med NEJM 1986;315:1046-51.

11. Bache RJ. Vasodilator reserve: A functional assessment of coronary health. Circulation 1998;98:1257-60.

12. Schachinger V, Britten MB, Zeiher AM. Prognostic impact of coronary vasodilator dysfunction on adverse long-term outcome of coronary heart disease. Circulation 2000;101:1899-906.

13. Gould KL. Johnson NP coronary physiology beyond coronary flow reserve in microvascular angina JACC state-of-the-art review. JACC 2018;72:2642-62.

14. Murthy VL, Naya M, Foster CR, Hainer J, Gaber M, Di Carli G, Blankstein R, Dorbala S, Sitek A, Pencina MJ, Di Carli MF. Improved cardiac risk assessment with noninvasive measures of coronary flow reserve. Circulation 2011;124:2215-24.

15. Ziadi MC, deKemp RA, Williams KA, et al. Impaired myocardial flow reserve on rubidium-82 positron emission tomography imaging predicts adverse outcomes in patients assessed for myocardial ischemia. J Am Coll Cardiol 2011;58:740-8.

16. Taqueti VR, Hachamovitch R, Murthy VL, Naya M, Foster CR, Hainer J, Dorbala S, Blankstein R, Di Carli MF. Global coronary flow reserve is associated with adverse cardiovascular events independently of luminal angiographic severity and modifies the effect of early revascularization. Circulation 2015;131:19-27.

17. Camici PG, Crea F. Coronary microvascular dysfunction. N Engl J Med 2007;356:830-40. https://doi.org/10.1056/NEJMra061889.

18. Taqueti VR, Di Carli MF. Coronary microvascular disease pathogenic mechanisms and therapeutic options: JACC state-ofthe-art review. J Am Coll Cardiol 2018;72:2625-41. https://doi. org/10.1016/j.jacc.2018.09.042.

19. Boden WE, O'Rourke RA, Teo KT, Hartigan PM, et al. Optimal medical therapy with or without PCI for stable coronary disease. New Engl J Med 2007;356:1503-16.

20. Shaw LJ, Berman DS, Maron DJ, Mancini GBJ, Hayes SW, Hartigan PM, Weintraub WS, O'Rourke RA, Dada M, Spertus JA, Chaitman BR, Friedman J, Slomka P, Heller GV, Germano G, Gosselin G, Berger P, Kostuk WJ, Schwartz RG, Knudtson M, Veledar E, Bates ER, McCallister B, Teo KK. Boden WE for the COURAGE Investigators. Optimal medical therapy with or without percutaneous intervention to reduce ischemic burden: Results from the Clinical Outcomes Utilizing Aggressive Drug Evaluation (COURAGE) trial nuclear substudy. Circulation 2008;117:128391.

21. Gould KL. Reversal of coronary atherosclerosis: Clinical promise as the basis for the non-invasive management of coronary artery disease. Circulation 1994;90:1558-71.

22. Gould KL, Ornish D, Scherwitz L, et al. Changes in myocardial perfusion abnormalities by positron emission tomography after long-term, intense risk factor modification. JAMA 1995;274:894901. https://doi.org/10.1001/jama.1995.03530110056036. 
23. Schwartz RG, Pearson TA. Can single photon emission computed tomography myocardial perfusion imaging monitor the potential benefit of aggressive treatment of hyperlipidemia? (editorial point of view). J Nucl Cardiol 1997;4:555-68.

24. Schwartz RG, Pearson TA, Kalaria VG, Mackin ML, Williford DJ, Awasthi A, Shah A. Prospective serial evaluation of myocardial perfusion and lipids during the first six months of pravastatin therapy: Coronary artery disease regression single photon emission computed tomography monitoring trial. J Am Coll Cardiol 2003;42:600-10.

25. Ladenheim ML, Pollock BH, Rozanski, Berman DS, Staniloff HM, Forrester JS, Diamond GA. Extent and severity of myocardial hypoperfusion as predictors of prognosis in patients with suspected coronary artery disease. J Am Coll Cardiol 1986;7:464-71.

26. Cerqueira MD, Weissman NJ, Dilsizian V, et al. Standardized myocardial segmentation and nomenclature for tomographic imaging of the heart: A statement for healthcare professionals from the Cardiac Imaging Committee of the Council on Clinical Cardiology of the American Heart Association. J Nucl Cardiol 2002;9:240-5. https://doi.org/10.1067/mnc.2002.123122.
27. Tilkemeier PL, Bourque J, Doukky R, et al. ASNC imaging guidelines for nuclear cardiology procedures. J Nucl Cardiol 2017;24:2064-128. https://doi.org/10.1007/s12350-017-1057-y.

28. Shepherd J, Cobbe SM, Ford I, et al. Prevention of coronary heart disease with pravastatin in men with hypercholesterolemia. West of Scotland Coronary Prevention Study Group. N Engl J Med 1995;333:1301-7.

29. Maron DJ, Hochman JS, Reynolds HR, et al. Initial invasive or conservative strategy for stable coronary disease. N Engl J Med 2020;382:1395-407.

30. Renaud JM, Premaratne, M, Villeneuve MC, Finnerty V, Harel, F, Heinonen, T, Tardif JC, Ruddy TD, deKemp R. MITNEC JNC 2020

31. Renaud JM, Mylonas I, McArdle B, et al. Clinical interpretation standards and quality assurance for the multicenter PET/CT trial rubidium-ARMI. J Nucl Med 2014;55:58-64.

Publisher's Note Springer Nature remains neutral with regard to jurisdictional claims in published maps and institutional affiliations. 